\title{
Searches for point-like sources of cosmic neutrinos with 13 years of ANTARES data
}

\author{
Giulia Illuminati $^{a, b, *}$ on behalf of the ANTARES Collaboration \\ (a complete list of authors can be found at the end of the proceedings) \\ ${ }^{a}$ INFN - Sezione di Bologna, Viale Berti-Pichat 6/2, 40127 Bologna, Italy \\ ${ }^{b}$ Dipartimento di Fisica e Astronomia dell'Università, Viale Berti Pichat 6/2, 40127 Bologna, Italy \\ E-mail: giulia.illuminati3@unibo.it
}

The main goal of the ANTARES neutrino telescope is the identification of neutrinos from astrophysical sources. Thanks to its location in the Northern hemisphere, ANTARES can rely on an advantageous view of the Southern Sky, in particular for neutrino energies below $100 \mathrm{TeV}$. This feature, combined with a very good angular resolution for high-quality selected events, makes the telescope an excellent tool to test for the presence of point-like sources, especially of Galactic origin. In ANTARES, track-like events (mainly resulting from $v_{\mu}$ charged current $-\mathrm{CC}-$ interactions) are reconstructed with a median angular resolution of $0.4^{\circ}$ while for shower-like events (mainly coming from $v_{e} \mathrm{CC}$ and all-flavour neutral current - $\mathrm{NC}$ - interactions) a median angular resolution of $3^{\circ}$ is achieved. The ANTARES Collaboration published the result of the search for cosmic point-like neutrino sources using track-like and shower-like events collected during nine years of data taking [Phys. Rev. D 96 (2017) 082001]. In this contribution, the update to this analysis using a total of 13 years of data recorded between early 2007 and early 2020 (3845 days of livetime) is presented. Moreover, the results of the dedicated searches for neutrino candidates from the tidal disruption events AT2019dsg and AT2019fdr, recently indicated as the most likely counterparts of two high-energy IceCube neutrinos, IC191001A and IC200530A, are reported.

$37^{\text {th }}$ International Cosmic Ray Conference (ICRC 2021)

July 12 th - 23rd, 2021

Online - Berlin, Germany

\footnotetext{
${ }^{*}$ Presenter
} 


\section{Introduction}

ANTARES is a high-energy neutrino telescope made of a three-dimensional array of 885 photomultiplier tubes (PMTs), deployed at $2500 \mathrm{~m}$ below the surface of the Mediterranean Sea, $40 \mathrm{~km}$ off-shore from Toulon, France [1]. Being located in the Northern hemisphere and immersed in sea water, ANTARES can count on a clear visibility of the Southern Sky, and thus of the Galactic Centre, and on a very good angular resolution. This makes it an excellent tool in the search for point-like sources, especially of Galactic origin, and a valid instrument to follow-up the the recent evidence of neutrino emission from the blazar TXS 0506+056 [2,3] and possibly from tidal disruption events (TDEs) AT2019dsg and AT2019fdr [4, 5], reported by the IceCube Collaboration. In these proceedings, the results of several searches for point-like sources using 13 years of ANTARES data are presented. This analysis updates the 9-year ANTARES point-like source search [6], and the subsequent 11-year analysis [7], employing a total of 13 years of data. Moreover, recently improved calibrations have been used to reconstruct all the employed ANTARES events, yielding slightly different values of the reconstructed direction and of the quality parameters associated to each event. The employed data set includes 10162 tracks and 225 showers recorded in ANTARES between January 29, 2007 and February 29, 2020 (3845 day livetime). Track-like events are mostly the result of the passage in water of relativistic muons produced in charged current (CC) interactions of muon neutrinos, while neutral current (NC) interactions, and $v_{e}$ and $v_{\tau} \mathrm{CC}$ interactions mainly induce shower-like events. The events have been selected using the criteria defined in [6], optimised to minimise the neutrino flux needed for a $5 \sigma$ discovery of a point-like source emitting with a $\propto E^{-2.0}$ spectrum. The selected tracks are reconstructed with a median angular resolution better than $0.4^{\circ}$ for energies above $10 \mathrm{TeV}$, while a median angular accuracy of $\sim 3^{\circ}$ is achieved for showers.

\section{Search Method}

The search for spatial clustering of events above the known background expectation relies on an unbinned maximum likelihood ratio approach. The employed likelihood is defined as

$$
\log \mathscr{L}=\sum_{j} \sum_{i \in j} \log \left[\frac{\mu_{\mathrm{sig}}^{j}}{\mathcal{N}^{j}} \mathcal{S}_{i}^{j}+\frac{\mathcal{N}^{j}-\mu_{\mathrm{sig}}^{j}}{\mathcal{N}^{j}} \mathcal{B}_{i}^{j}\right],
$$

where, $j$ denotes the sample (tr for tracks, $s h$ for showers), $i$ indicates the event of the sample $j$, $\mu_{\text {sig }}^{j}$ is the number of signal events fitted in the $j$ sample, $\mathcal{N}^{j}$ is the total number of events in the $j$ sample, and $\mathcal{S}_{i}^{j}$ and $\mathcal{B}_{i}^{j}$ are the values of the signal and background PDFs for the event $i$ in the sample $j$. The signal and background PDFs are given by the product of a directional and an energy terms. The same definition of the PDFs used in the previous analyses [6, 7] is employed. In the likelihood maximization, the number of signal events $\mu_{\text {sig }}^{\mathrm{tr}}$ and $\mu_{\text {sig }}^{\text {sh }}$ are free parameters. Moreover, the position in the sky of the source is either kept fixed or allowed to be fitted within specific limits depending on the type of search (see Section 3). The signal likeness of a cluster is determined by a test statistic computed as 


$$
Q=\log \mathscr{L}^{\max }-\log \mathscr{L}^{\mathrm{bkg}},
$$

where $\mathscr{L}^{\max }$ and $\mathscr{L}^{\text {bkg }}$ are the values of the likelihood defined in Equation 1 calculated using the best-fit values of the free parameters and for the background-only case ( $\left.\mu_{\text {sig }}^{\mathrm{tr}}=\mu_{\mathrm{sig}}^{\mathrm{sh}}=0\right)$, respectively. In order to calculate the significance of any observation, the observed $Q$ is compared to the test statistic distribution obtained in background-only pseudo-experiments (PEs) - pseudo-data sets of data randomised in right ascension. The fraction of $Q$ values which are larger than the observed $Q$ gives the significance (p-value) of the observation.

\section{Searches and Results}

Two searches for astrophysical neutrino sources are performed using the whole described data set: a scan over the whole ANTARES visible sky, and a survey of 121 astrophysical candidates. These approaches are described below together with the corresponding results. Moreover, the results of dedicated searches for cosmic neutrinos associated with AT2019dsg and AT2019fdr are reported. In this case, only the events detected by ANTARES from the day of the discovery of each TDE (2019 April 9 and 2019 April 27, respectively), until the day of the last available fullycalibrated ANTARES data, 2020 February 29, have been used, corresponding to a livetime of 315 and 298 days, respectively. Additional information on the search from the TDEs can be found in [8].

\subsection{Full-Sky Search}

In the full-sky search, the ANTARES visible sky is scanned to look for the most significant excess of signal events, without making any assumption about the source position. To this purpose, the $Q$-value defined in equation (2) is evaluated in steps of $1^{\circ} \times 1^{\circ}$ over the whole scanned region, with the location of the fitted cluster being left free to vary within these boundaries. The most significant cluster of this search, i.e. the cluster with lowest pre-trial p-value, is found at a right ascension of $\alpha=39.6^{\circ}$ and a declination of $\delta=11.1^{\circ}$ with a pre-trial p-value of $6.8 \times 10^{-6}(4.3 \sigma)$, corresponding to a post-trial p-value of $48 \%$, and a number of fitted signal events $\hat{\mu}_{\text {sig }}=6.3$. The hotspot is at $1^{\circ}$ angular distance from the source J0242+1101 (also known as PKS 0239+108)- a radio-bright blazar investigated in the candidate-list search. The second most significant location is found at equatorial coordinates $\left(\alpha=343.8^{\circ}, \delta=23.5^{\circ}\right)$ and corresponds to the full-sky hotspot found in the 11-year ANTARES point-like source search [7]. As already highlighted in [7], this location is in the vicinity of the IceCube high-energy muon track with ID 3 [9]. Moreover, it is located within $1^{\circ}$ from the blazar MG3 J225517+2409. The blazar was in flaring state during the IceCube neutrino arrival time [10]. An analysis of the space and time correlation between the ANTARES and IceCube close to the blazar and of the gamma-ray flux measured by Fermi has been performed by ANTARES, leading to a chance probability for the association of $1.9 \sigma$ [10]. The position of the most and second most significant clusters of the full-sky search together with the pre-trial p-values found at each direction of the ANTARES visible sky in shown in Figure 1. The distribution of the ANTARES events around the two best locations is shown in Figure 2. 

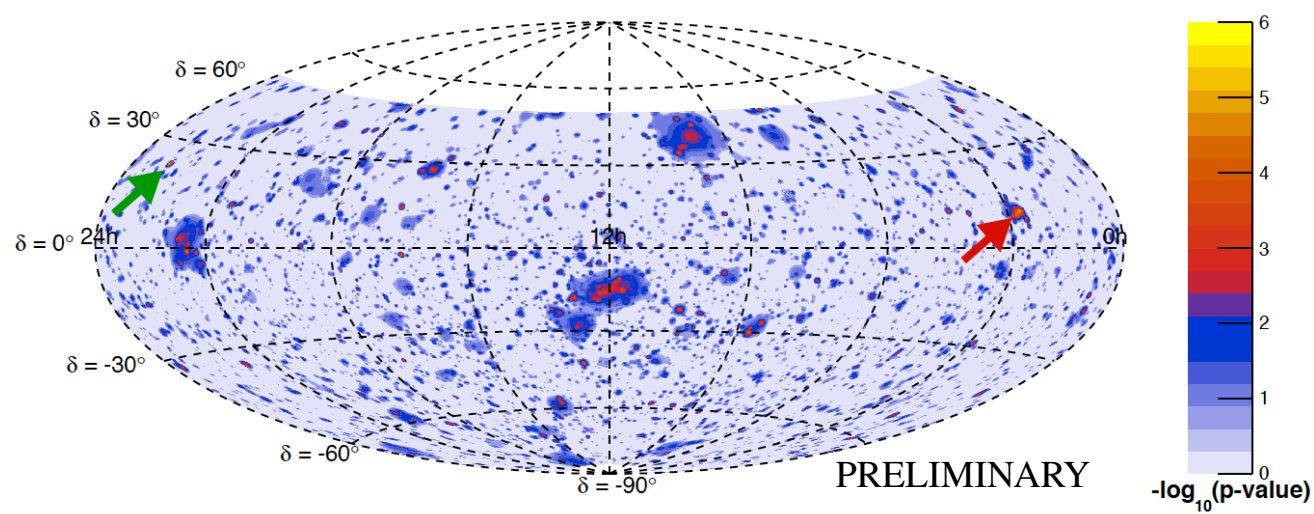

Figure 1: Sky map in equatorial coordinates of pre-trial p-values for a point-like source of the ANTARES visible sky. The location of the most (second most) significant cluster of the full-sky search is indicated by the red (green) arrow.

\subsection{Candidate-List Search}

In the candidate-list search, a pre-selected list of potential neutrino sources is investigated to look for an excess of neutrino events. In the likelihood maximization, the equatorial coordinates of the source are kept fixed at the known value, and only the number of signal events is left as free parameter. In case of null observation, upper limit on their neutrino fluxes are determined. The candidate list employed in this analysis updates the one used in [7] by including the following sources:

- two new sources reported in the TeVCat catalog [11] and detected after June 2019 (date of the latest update);

- the sources recently detected by LHAASO [12], with a declination below $42^{\circ}$. In case of spatial overlap (angular distance smaller than $0.5^{\circ}$ ) with candidates already in the list, the already investigated candidates have been replaced with the LHAASO sources;

- the Galactic sources with emission above $56 \mathrm{TeV}$ detected by HAWC [13], not in coincidence with sources already in the list;

- the blazar TXS 0506+056;

- the Seyfert II galaxy NGC 1068, i.e. the most significant source in the IceCube Northern catalog, coincident with the IceCube Northern-sky hotspot [14];

- the radio-bright blazar J0242+1101, for which a hint of a spatial and temporal association between ANTARES neutrinos, a radio flare, and a gamma-ray flare observed by Fermi has been found [15].

The list of the 121 analysed candidates, together with the obtained results at each location, is reported in Table 1. The most signal-like cluster is found at the location of J0242+1101, with a pre-trial p-value of $6.7 \times 10^{-5}(3.8 \sigma)$. The post-trial significance, calculated taking into account that other 120 locations have been investigated in this search, is of $2.4 \sigma$. The source is located at $1^{\circ}$ angular distance from the full-sky hotspot, as shown in Figure 2. J0242+1101 is followed in significance by TXS 0506+056 (2.8 $\sigma$ pre-trial), HESSJ0632+057 (2.1 $\sigma$ pre-trial) and the Galactic 
Centre ( $2.0 \sigma$ pre-trial). The $90 \%$ C.L. limits on the one-flavour neutrino flux normalization, $\Phi_{0}$, where the neutrino flux has been parameterised as $\Phi_{v}=\Phi_{0}\left(\frac{E_{v}}{1 \mathrm{GeV}}\right)^{-2}$, are shown in Figure 3 as a function of the declination for the 121 investigated candidates.

Table 1: List of analysed astrophysical objects. Reported are the source's name, equatorial coordinates $(\delta, \alpha)$, best-fit number of signal events $\hat{\mu}_{\text {sig }}$, pre-trial p-value and $90 \%$ C.L. upper limits on the flux normalization factor for a $E^{-2.0}$

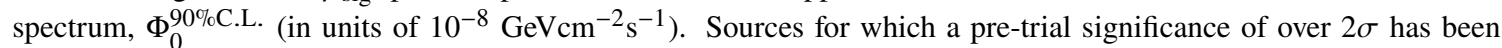
obtained are highlighted in bold. Dashes (-) in the fitted number of source events and pre-trial p-value indicate sources with null fitted signal.

\begin{tabular}{|c|c|c|c|c|c|c|c|c|c|c|c|}
\hline Name & $\delta\left[^{\circ}\right]$ & $\alpha\left[^{\circ}\right]$ & $\hat{\mu}_{\text {sig }}$ & p-value & $\Phi_{0}^{90 \% \text { C.L. }}$ & Name & $\delta\left[^{\circ}\right]$ & $\alpha\left[^{\circ}\right]$ & $\hat{\mu}_{\text {sig }}$ & p-value & $\Phi_{0}^{90 \% \text { C.I }}$ \\
\hline LHA120-N-157B & -69.16 & 84.43 & - & - & 0.43 & LHAASOJ1849-0003 & -0.05 & 282.35 & - & - & 0.67 \\
\hline HESSJ1356-645 & -64.50 & 209.00 & 0.7 & 0.16 & 0.73 & NGC1068 & -0.01 & 40.67 & - & - & 0.82 \\
\hline PSRB1259-63 & -63.83 & 195.70 & - & - & 0.44 & 2HWCJ1852+013* & 1.38 & 283.01 & - & - & 0.69 \\
\hline HESSJ1303-631 & -63.20 & 195.75 & - & - & 0.44 & W44 & 1.38 & 284.04 & - & - & 0.69 \\
\hline RCW86 & -62.48 & 220.68 & - & - & 0.45 & PKS0736+017 & 1.62 & 114.83 & - & - & 0.68 \\
\hline HESSJ1507-622 & -62.34 & 226.72 & - & - & 0.45 & RGBJ0152+017 & 1.79 & 28.17 & - & - & 0.68 \\
\hline HESSJ1458-608 & -60.88 & 224.54 & - & - & 0.48 & 2HWCJ1902+048* & 4.86 & 285.51 & _- & _- & 0.69 \\
\hline ESO139-G12 & -59.94 & 264.41 & - & - & 0.45 & SS433 & 4.98 & 287.96 & - & - & 0.69 \\
\hline MSH15-52 & -59.16 & 228.53 & - & - & 0.45 & TXS0506+056 & 5.70 & 77.35 & 2.9 & 0.0020 & 2.13 \\
\hline HESSJ1503-582 & -58.74 & 226.46 & - & - & 0.45 & HESSJ0632+057 & 5.81 & 98.24 & 1.7 & 0.017 & 1.70 \\
\hline HESSJ1023-575 & -57.76 & 155.83 & - & - & 0.66 & LHAASOJ1908+0621 & 6.35 & 287.05 & - & - & 0.70 \\
\hline CirX-1 & -57.17 & 230.17 & - & - & 0.69 & 2HWCJ1829+070 & 7.03 & 277.34 & - & - & 0.69 \\
\hline SNRG327.1-01.1 & -55.08 & 238.65 & - & - & 0.56 & B1030+074 & 7.19 & 158.39 & - & - & 0.69 \\
\hline HESSJ1614-518 & -51.82 & 243.58 & - & - & 0.45 & 2HWCJ1907+084* & 8.50 & 286.79 & - & - & 0.70 \\
\hline HESSJ1616-508 & -50.97 & 243.97 & - & - & 0.44 & OT081 & 9.65 & 267.89 & 1.9 & 0.051 & 1.45 \\
\hline PKS2005-489 & -48.82 & 302.37 & 1.2 & 0.14 & 0.77 & HESSJ1912+101 & 10.15 & 288.21 & - & - & 0.87 \\
\hline GX339-4 & -48.79 & 255.70 & - & - & 0.45 & PKS $1502+106$ & 10.52 & 226.10 & - & - & 0.71 \\
\hline HESSJ1632-478 & -47.82 & 248.04 & - & - & 0.56 & J0242+1101 & 11.02 & 40.62 & 6.1 & 0.000067 & 2.77 \\
\hline RXJ0852.0-4622 & -46.37 & 133.00 & - & - & 0.45 & RBS0723 & 11.56 & 131.80 & - & - & 0.85 \\
\hline HESSJ1641-463 & -46.30 & 250.26 & 1.0 & 0.13 & 0.76 & 2HWCJ1914+117 & 11.72 & 288.68 & - & - & 0.92 \\
\hline VelaX & -45.60 & 128.75 & 1.3 & 0.085 & 0.84 & 2HWCJ1921+131 & 13.13 & 290.30 & - & - & 0.71 \\
\hline PKS0537-441 & -44.08 & 84.71 & 1.3 & 0.094 & 0.85 & W51C & 14.19 & 290.75 & - & - & 0.71 \\
\hline CentaurusA & -43.02 & 201.36 & 0.9 & 0.086 & 0.84 & $2 \mathrm{HWCJ} 0700+143$ & 14.32 & 105.12 & - & - & 0.71 \\
\hline PKS1424-418 & -42.10 & 216.98 & - & - & 0.47 & VERJ0648+152 & 15.27 & 102.20 & - & - & 0.72 \\
\hline 1ES2322-409 & -40.66 & 351.20 & - & - & 0.51 & 2HWCJ0819+157 & 15.79 & 124.98 & - & - & 0.75 \\
\hline RXJ1713.7-3946 & -39.75 & 258.25 & - & - & 0.47 & $3 \mathrm{C} 454.3$ & 16.15 & 343.50 & - & - & 0.73 \\
\hline PKS1440-389 & -39.14 & 220.99 & - & - & 0.48 & PKS0235+164 & 16.61 & 39.66 & - & - & 0.72 \\
\hline PKS0426-380 & -37.93 & 67.17 & - & - & 0.49 & Geminga & 17.77 & 98.47 & - & - & 0.97 \\
\hline PKS1454-354 & -35.67 & 224.36 & 0.9 & 0.16 & 0.88 & LHAASOJ1929+1745 & 17.75 & 292.25 & - & - & 0.83 \\
\hline PKS0625-35 & -35.49 & 96.78 & - & - & 0.51 & RGBJ2243+203 & 20.35 & 340.98 & 1.3 & 0.033 & 1.68 \\
\hline TXS1714-336 & -33.70 & 259.40 & - & - & 0.71 & VERJ0521+211 & 21.21 & 80.44 & - & - & 1.05 \\
\hline SwiftJ1656.3-3302 & -33.04 & 254.07 & - & - & 0.76 & $4 C+21.35$ & 21.38 & 186.23 & - & - & 0.77 \\
\hline PKS0548-322 & -32.27 & 87.67 & - & - & 0.53 & Crab & 22.01 & 83.63 & - & - & 0.75 \\
\hline H2356-309 & -30.63 & 359.78 & - & - & 0.56 & IC443 & 22.50 & 94.21 & - & - & 0.76 \\
\hline PKS2155-304 & -30.22 & 329.72 & - & - & 0.55 & S20109+22 & 22.74 & 18.02 & - & - & 0.76 \\
\hline HESSJ1741-302 & -30.20 & 265.25 & 1.1 & 0.088 & 1.06 & B1422+231 & 22.93 & 216.16 & - & - & 0.76 \\
\hline PKS1622-297 & -29.90 & 246.50 & - & - & 0.72 & PKS $1424+240$ & 23.79 & 216.75 & - & - & 0.77 \\
\hline GalacticCentre & -29.01 & 266.42 & 2.2 & 0.021 & 1.36 & 2HWCJ1938+238 & 23.81 & 294.74 & - & - & 0.77 \\
\hline Terzan5 & -24.90 & 266.95 & - & - & 0.76 & 2HWCJ1949+244 & 24.46 & 297.42 & 0.6 & 0.12 & 1.32 \\
\hline 1ES1101-232 & -23.49 & 165.91 & - & - & 0.59 & MS1221.8+2452 & 24.61 & 186.10 & - & - & 0.78 \\
\hline PKS0454-234 & -23.43 & 74.27 & - & - & 0.59 & PKS1441+25 & 25.03 & 220.99 & - & - & 0.89 \\
\hline W28 & -23.34 & 270.43 & - & - & 0.87 & $1 \mathrm{ES} 0647+250$ & 25.05 & 102.69 & - & - & 1.00 \\
\hline PKS1830-211 & -21.07 & 278.42 & - & - & 0.60 & S31227+25 & 25.30 & 187.56 & - & - & 0.78 \\
\hline eHWCJ1809-193 & -19.34 & 272.46 & - & - & 0.59 & WComae & 28.23 & 185.38 & - & - & 0.81 \\
\hline SNRG015.4+00.1 & -15.47 & 274.52 & - & - & 0.62 & LHAASOJ1956+2845 & 28.75 & 299.05 & - & - & 0.82 \\
\hline LS5039 & -14.83 & 276.56 & - & - & 0.62 & TON0599 & 29.24 & 179.88 & - & - & 0.82 \\
\hline QSO1730-130 & -13.10 & 263.30 & 1.1 & 0.12 & 1.09 & 2HWCJ1953+294 & 29.48 & 298.26 & - & - & 0.82 \\
\hline LHAASOJ1825-1326 & -13.45 & 276.45 & - & - & 0.61 & 1ES1215+303 & 30.10 & 184.45 & - & - & 0.83 \\
\hline HESSJ1813-126 & -12.68 & 273.34 & - & - & 0.61 & 1ES1218+304 & 30.19 & 185.36 & - & - & 0.83 \\
\hline 1ES0347-121 & -11.99 & 57.35 & - & - & 0.61 & HESSJ1746-308 & 30.84 & 266.57 & - & - & 1.15 \\
\hline PKS0727-11 & -11.70 & 112.58 & 1.6 & 0.026 & 1.48 & 2HWCJ1040+308 & 30.87 & 160.22 & - & - & 0.84 \\
\hline HESSJ1828-099 & -9.99 & 277.24 & 0.5 & 0.15 & 1.05 & B21811+31 & 31.74 & 273.40 & - & - & 0.85 \\
\hline HESSJ1831-098 & -9.90 & 277.85 & - & - & 0.62 & B21420+32 & 32.39 & 215.63 & - & - & 0.92 \\
\hline HESSJ1834-087 & -8.76 & 278.69 & - & - & 0.64 & 2HWCJ2006+341 & 34.18 & 301.55 & - & - & 0.88 \\
\hline PKS1406-076 & -7.90 & 212.20 & - & - & 0.63 & S30218+35 & 35.94 & 35.27 & - & - & 0.91 \\
\hline QSO2022-077 & -7.60 & 306.40 & 1.0 & 0.070 & 1.27 & LHAASOJ2018+3651 & 36.85 & 304.75 & 0.5 & 0.14 & 1.44 \\
\hline HESSJ1837-069 & -6.95 & 279.41 & - & - & 0.77 & MilagroDiffuse & 38.00 & 305.00 & 0.9 & 0.11 & 1.59 \\
\hline $3 C 279$ & -5.79 & 194.05 & 1.2 & 0.074 & 1.28 & Markarian421 & 38.19 & 166.08 & - & - & 0.93 \\
\hline LHAASOJ1839-0545 & -5.75 & 279.95 & - & - & 0.70 & B32247+381 & 38.43 & 342.53 & - & - & 0.93 \\
\hline 2HWCJ1309-054 & -5.49 & 197.31 & - & - & 0.66 & LHAASOJ2032+4102 & 41.05 & 308.05 & - & - & 0.96 \\
\hline LHAASOJ1843-0338 & -3.65 & 280.75 & - & - & 0.68 & & & & & & \\
\hline
\end{tabular}





Figure 2: Distribution of the ANTARES events close to the best and second best locations found in each search in the $(\alpha, \delta)$ (RA, DEC) coordinates. The left plot is centered at the position of the full-sky hotspot (magenta star), which is at $1^{\circ}$ angular distance from the source $\mathrm{J} 0242+1101$, i.e. the most significant source of the candidate-list search, indicated by the orange star. The central plot shows the cluster of events around TXS 0506+056. The right plot is centered at the location of the second most significant cluster of the full-sky search (magenta star), and shows also the position of MG3 J225517+2409 (orange star) and of the IceCube high-energy muon track (grey star). In each plot, the inner (outer) solid green line depicts the one (five) degree distance from the position of the best-fit or known location. The red points denote shower-like events, whereas the blue points indicate track-like events. The dashed circles around the events indicate the angular error estimate. Different tones of red and blue correspond to the values assumed by the energy estimators: the number of hits (shower-like events) and the $\rho$ parameter (track-like events) as shown in the legend. Refer to [6] for further details on the energy estimators.

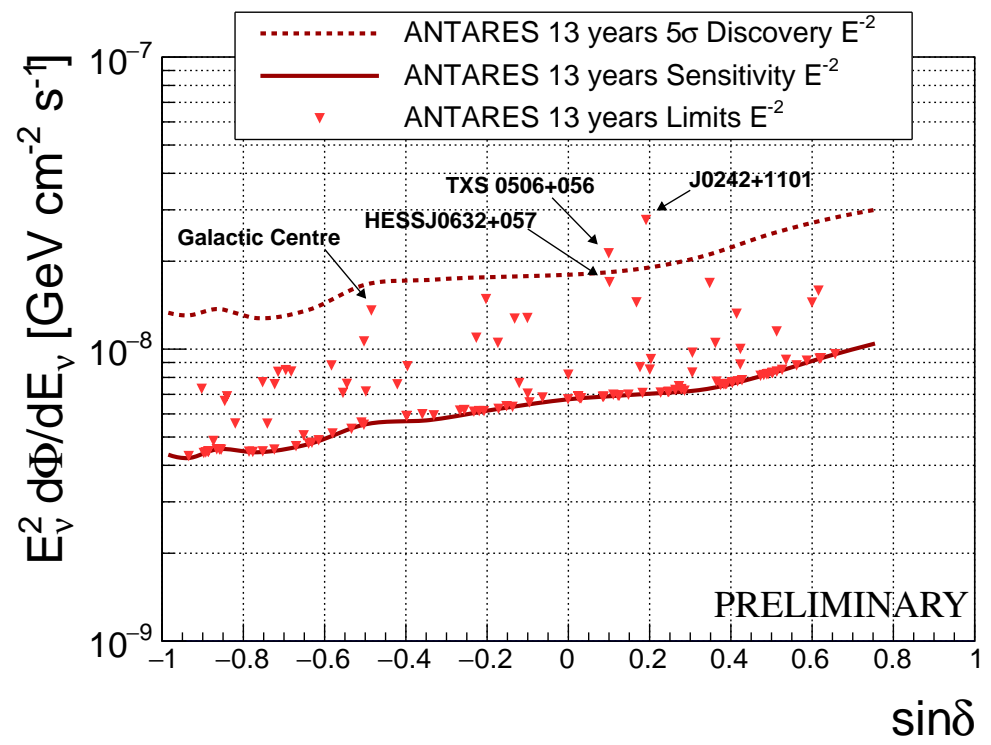

Figure 3: 90\% C.L. upper limits (triangles) on the one-flavour neutrino flux normalization for the investigated astrophysical candidates as a function of the source declination assuming a $E^{-2.0}$ spectrum. The name of the sources for which a pre-trial significance of over $2 \sigma$ has been obtained is reported beside the arrow pointing to the corresponding upper limit. The solid (dashed) line shows the $90 \%$ C.L. median sensitivity (50\% $5 \sigma$ discovery flux) of the analysis. 


\subsection{Dedicated search at the TDEs AT2019dsg and AT2019fdr}

The location of AT2019dsg and AT2019fdr has been investigated using the same approach described in Section 3.2, and employing only the ANTARES data collected since the discovery of each TDEs. An unbroken power law neutrino spectrum, $\propto E^{-\gamma}$, is assumed in this analysis for the signal emission, with $\gamma$ being one of the three tested spectral indices, $\gamma=2.0, \gamma=2.5$, or $\gamma=3.0$. The distribution of the ANTARES events close to the location of the sources is shown in Figure 4. In both cases, only one event has been detected within $5^{\circ}$ from the TDE. The event close to AT2019dsg (AT2019fdr) is a track-like event, located at a distance of $1.7^{\circ}\left(2.3^{\circ}\right)$ from the source, and was registered on February 11, 2020 (September 25, 2019). Table 2 summarises the results of the searches in terms of best-fit number of signal events and p-value, and reports the upper limits on the one-flavour neutrino flux normalisation, $\Phi_{0}$, for three values of $\gamma$, where the neutrino flux has been parameterised as $\frac{d N_{v}}{d E_{v}}=\Phi_{0}\left(\frac{E_{v}}{1 \mathrm{GeV}}\right)^{-\gamma}$. Moreover, upper limits are set on the one-flavour neutrino fluence, $\mathcal{F}$, defined as the integral in time and energy of the neutrino energy flux, $\mathcal{F}=\int_{t_{\min }}^{t_{\max }} \int_{E_{\min }}^{E_{\max }} E_{\nu} \cdot \frac{d N_{v}}{d E_{v}} d E_{v} d t=\Delta t \cdot \Phi_{0} \cdot \int_{E_{\min }}^{E_{\max }} E_{\nu} \cdot\left(\frac{E_{v}}{1 \mathrm{GeV}}\right)^{-\gamma} d E_{\nu}$, with $E_{\min }$ and $E_{\max }$ being the boundaries of the energy range containing $90 \%$ of the expected signal events for the given $\gamma$, and $\Delta t$ is the livetime of the search.


Figure 4: Distribution of ANTARES events in equatorial coordinates around the position of AT2019dsg (left) and AT2019fdr (right). The orange lines depict the one and five degree distance from the source position, indicated as a grey star. Only track-like events (blue points) have been detected within $5^{\circ}$ from the location of the sources. The dashed circles around the events indicate the angular error estimate on the track reconstructed direction.

\section{Conclusions}

The results of various searches for point-like sources using events detected by the ANTARES telescope during 13 years of data taking have been presented. The searches include a scan over the whole ANTARES visible sky, an investigation of 121 astrophysical candidates, and a dedicated analysis of the direction of tidal disruption events AT2019dsg and AT2019fdr. No significant evidence of cosmic neutrino sources has been found. The full-sky hotspot $\left(\alpha=39.6^{\circ}, \delta=11.1^{\circ}\right)$ has a pre-trial p-value of $6.8 \times 10^{-6}(4.3 \sigma)$, corresponding to $48 \%$ post-trial p-value. Four sources investigated in the candidate-list search show a pre-trial significance above $2 \sigma: \mathrm{J} 0242+1101(3.8 \sigma)$, 
Table 2: Results of the search at the location of AT2019dsg and AT2019fdr in terms of best-fit number of signal events $\hat{\mu}_{\text {sig }}$, p-value, 90\% C.L. sensitivity and upper limits on the one-flavour neutrino flux normali-

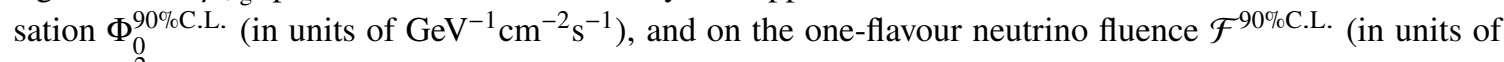
$\mathrm{GeVcm}^{-2}$ ), for different values of the spectral index $\gamma$. The boundaries, $E_{\min }$ and $E_{\max }$, of the energy range containing $90 \%$ of the expected signal events, employed in the calculation of the fluence, are listed in the last column.

\begin{tabular}{|c|c|c|c|c|c|c|c|c|}
\hline \multicolumn{2}{|l|}{ Source } & \multicolumn{7}{|c|}{ Results } \\
\hline \multirow[t]{2}{*}{ Name } & \multirow[t]{2}{*}{$\gamma$} & \multirow[t]{2}{*}{$\hat{\mu}_{\text {sig }}$} & \multirow[t]{2}{*}{ p-value } & \multicolumn{2}{|c|}{$\Phi_{0}^{90 \% \text { C.L. }}$} & \multirow{2}{*}{\multicolumn{2}{|c|}{$\begin{array}{c}\mathcal{F}^{90 \% \text { C.L. }} \\
\text { sensitivity }\end{array}$}} & \multirow{2}{*}{$\log \left(\frac{E_{\min }}{\mathrm{GeV}}\right)-\log \left(\frac{E_{\max }}{\mathrm{GeV}}\right)$} \\
\hline & & & & sensitivity & limit & & & \\
\hline \multirow[t]{3}{*}{ AT2019dsg } & 2.0 & $<0.1$ & $12.4 \%$ & $7.3 \times 10^{-8}$ & $1.0 \times 10^{-7}$ & 14 & 19 & $3.6-6.6$ \\
\hline & 2.5 & 0.2 & $10.2 \%$ & $1.5 \times 10^{-5}$ & $2.2 \times 10^{-5}$ & 29 & 43 & $2.8-5.5$ \\
\hline & 3.0 & 0.7 & $8.9 \%$ & $1.2 \times 10^{-3}$ & $2.0 \times 10^{-3}$ & 230 & 380 & $2.1-4.7$ \\
\hline \multirow[t]{3}{*}{ AT2019fdr } & 2.0 & 0.5 & $6.7 \%$ & $8.5 \times 10^{-8}$ & $1.3 \times 10^{-7}$ & 15 & 23 & $3.6-6.6$ \\
\hline & 2.5 & 0.5 & $7.9 \%$ & $2.1 \times 10^{-5}$ & $3.0 \times 10^{-5}$ & 39 & 55 & $2.8-5.5$ \\
\hline & 3.0 & 0.6 & $9.1 \%$ & $2.0 \times 10^{-3}$ & $3.0 \times 10^{-3}$ & 360 & 540 & $2.1-4.7$ \\
\hline
\end{tabular}

located at $1^{\circ}$ angular distance from the full-sky hotspot, TXS 0506+056 $(2.8 \sigma)$, HESSJ0632+057 $(2.1 \sigma)$ and the Galactic Centre $(2.0 \sigma)$.

\section{References}

[1] ANTARES Collaboration, M. Ageron et al., Nucl. Instrum. Meth. A656 (2011) 11.

[2] IceCube Collaboration, M. G. Aartsen et al., Science 361 (2018) 147-151.

[3] IceCube, Fermi-LAT, MAGIC, AGILE, ASAS-SN, HAWC, HESS, INTEGRAL, Kanata, Kiso, Kapteyn, Liverpool Telescope, Subaru, Swift NuSTAR, VERITAS, VLA/17B-403 Collaboration, M. G. Aartsen et al., Science 361 (2018) eaat1378.

[4] R. Stein et al., Nature Astron. 5 (2021) 510-518.

[5] Zwicky Transient Facility (ZTF) and Global Relay of Observatories Watching Transients Happen (GROWTH) Collaboration, S. Reusch, GCN Circular 27872 (2020).

[6] ANTARES Collaboration, A. Albert et al., Phys. Rev. D96 (2017) 082001.

[7] ANTARES Collaboration, J. Aublin, G. Illuminati, and S. Navas, PoS(ICRC2019)920 (2020).

[8] ANTARES Collaboration, A. Albert et al., arXiv:2103.15526.

[9] IceCube Collaboration, M. G. Aartsen et al., Astrophys. J. 833 (2016) 3.

[10] ANTARES Collaboration, A. Albert et al., Astrophys. J. 911 (2021) 48.

[11] TevCat catalog, http://tevcat.uchicago.edu/.

[12] LHAASO Collaboration, Z. Cao et al., Nature 594 (2021) 33-36.

[13] HAWC Collaboration, A. U. Abeysekara et al., Phys. Rev. Lett. 124 (2020) 021102.

[14] IceCube Collaboration, M. G. Aartsen et al., Phys. Rev. Lett. 124 (2020) 051103.

[15] ANTARES Collaboration, G. Illuminati, PoS(ICRC2021)1137 (these proceedings). 


\section{Full Authors List: ANTARES Collaboration}

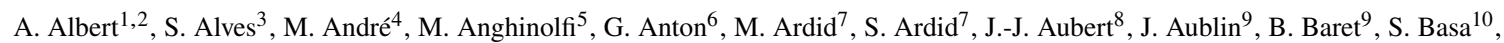

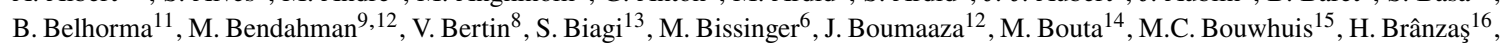
R. Bruijn ${ }^{15,17}$, J. Brunner ${ }^{8}$, J. Busto ${ }^{8}$, B. Caiffi ${ }^{\text {, A. Capone }}{ }^{18,19}$, L. Caramete ${ }^{16}$, J. Carr ${ }^{8}$, V. Carretero ${ }^{3}$, S. Celli ${ }^{18,19}$, M. Chabab ${ }^{20}$ T. N. Chau $^{9}$, R. Cherkaoui El Moursli ${ }^{12}$, T. Chiarusi ${ }^{21}$, M. Circella $^{22}$, A. Coleiro ${ }^{9}$, M. Colomer-Molla ${ }^{9,3}$, R. Coniglione ${ }^{13}$, P. Coyle ${ }^{8}$, A. Creusot $^{9}$, A. F. Díaz ${ }^{23}$, G. de Wasseige ${ }^{9}$, A. Deschamps ${ }^{24}$, C. Distefano ${ }^{13}$, I. Di Palma ${ }^{18,19}$, A. Domi ${ }^{15,17}$, C. Donzaud ${ }^{9,25}$, D. Dornic ${ }^{8}$, D. Drouhin ${ }^{1,2}$, T. Eberl ${ }^{6}$, T. van Eeden ${ }^{15}$, D. van Eijk ${ }^{15}$, N. El Khayati ${ }^{12}$, A. Enzenhöfer ${ }^{8}$, P. Fermani ${ }^{18,19}$, G. Ferrara $^{13}$ F. Filippini ${ }^{21,26}$, L.A. Fusco ${ }^{8}$, Y. Gatelet ${ }^{9}$, P. Gay ${ }^{27,9}$, H. Glotin ${ }^{28}$, R. Gozzini ${ }^{3}$, R. Gracia Ruiz ${ }^{15}$, K. Graf ${ }^{6}$, C. Guidi ${ }^{5}, 29$, S. Hallmann ${ }^{6}$, H. van Haren ${ }^{30}$, A.J. Heijboer ${ }^{15}$, Y. Hello ${ }^{24}$, J.J. Hernández-Rey ${ }^{3}$, J. Höß1 ${ }^{6}$, J. Hofestädt ${ }^{6}$, F. Huang ${ }^{8}$, G. Illuminati ${ }^{9}, 21,26$, C.W James ${ }^{31}$, B. Jisse-Jung ${ }^{15}$, M. de Jong ${ }^{15,32}$, P. de Jong ${ }^{15}$, M. Kadler ${ }^{33}$, O. Kalekin ${ }^{6}$, U. Katz ${ }^{6}$, N.R. Khan-Chowdhury ${ }^{3}$, A. Kouchner ${ }^{9}$, I. Kreykenbohm ${ }^{34}$, V. Kulikovskiy ${ }^{5,36}$, R. Lahmann ${ }^{6}$, R. Le Breton ${ }^{9}$, D. Lefèvre ${ }^{35}$, E. Leonora ${ }^{36}$, G. Levi ${ }^{21,26}$, M. Lincetto ${ }^{8}$, D. Lopez-Coto ${ }^{37}$, S. Loucatos ${ }^{38,9}$, L. Maderer ${ }^{9}$, J. Manczak $^{3}$, M. Marcelin ${ }^{10}$, A. Margiotta ${ }^{21,26}$, A. Marinelli ${ }^{39}$, J.A. Martínez-Mora ${ }^{7}$, K. Melis ${ }^{15,17}$, P. Migliozzi ${ }^{39}$, A. Moussa ${ }^{14}$, R. Muller ${ }^{15}$, L. Nauta ${ }^{15}$, S. Navas ${ }^{37}$, E. Nezri ${ }^{10}$, B. O' Fearraigh $^{15}$, A. Păun ${ }^{16}$, G.E. Păvălaş ${ }^{16}$, C. Pellegrino ${ }^{21,40,41}$, M. Perrin-Terrin ${ }^{8}$, V. Pestel ${ }^{15}$, P. Piattelli ${ }^{13}$, C. Pieterse ${ }^{3}$, C. Poirè ${ }^{7}$, V. Popa ${ }^{16}$, T. Pradier ${ }^{1}$, N. Randazzo ${ }^{36}$, S. Reck $^{6}$, G. Riccobene ${ }^{13}$, A. Romanov ${ }^{5,29}$, A. Sánchez-Losa ${ }^{3,22}$, F. Salesa Greus ${ }^{3}$, D. F. E. Samtleben ${ }^{15,32}$, M. Sanguineti ${ }^{5,29}$, P. Sapienza ${ }^{13}$, J. Schnabel ${ }^{6}$, J. Schumann ${ }^{6}$, F. Schüssler ${ }^{38}$, M. Spurio ${ }^{21,26}$, Th. Stolarczyk ${ }^{38}$, M. Taiuti ${ }^{5,29}$, Y. Tayalati ${ }^{12}$, S.J. Tingay ${ }^{31}$, B. Vallage ${ }^{38,9}$, V. Van Elewyck ${ }^{9,41}$, F. Versari ${ }^{21,26,9}$, S. Viola ${ }^{13}$, D. Vivolo ${ }^{39,43}$, J. Wilms ${ }^{34}$, S. Zavatarelli ${ }^{5}$, A. Zegarelli ${ }^{18,19}$, J.D. Zornoza ${ }^{3}$, and J. Zúñiga ${ }^{3}$

${ }^{1}$ Université de Strasbourg, CNRS, IPHC UMR 7178, F-67000 Strasbourg, France. ${ }^{2}$ Université de Haute Alsace, F-68100 Mulhouse, France. ${ }^{3}$ IFIC - Instituto de Física Corpuscular (CSIC - Universitat de València) c/ Catedrático José Beltrán, 2 E-46980 Paterna, Valencia, Spain. ${ }^{4}$ Technical University of Catalonia, Laboratory of Applied Bioacoustics, Rambla Exposició, 08800 Vilanova i la Geltrú, Barcelona, Spain. ${ }^{5}$ INFN - Sezione di Genova, Via Dodecaneso 33, 16146 Genova, Italy. ${ }^{6}$ Friedrich-Alexander-Universität Erlangen-Nürnberg, Erlangen Centre for Astroparticle Physics, Erwin-Rommel-Str. 1, 91058 Erlangen, Germany. ${ }^{7}$ Institut d'Investigació per a la Gestió Integrada de les Zones Costaneres (IGIC) - Universitat Politècnica de València. C/ Paranimf 1, 46730 Gandia, Spain. ${ }^{8}$ Aix Marseille Univ, CNRS/IN2P3, CPPM, Marseille, France. ${ }^{9}$ Université de Paris, CNRS, Astroparticule et Cosmologie, F-75013 Paris, France. ${ }^{10}$ Aix Marseille Univ, CNRS, CNES, LAM, Marseille, France. ${ }^{11}$ National Center for Energy Sciences and Nuclear Techniques, B.P.1382, R. P.10001 Rabat, Morocco. ${ }^{12}$ University Mohammed V in Rabat, Faculty of Sciences, 4 av. Ibn Battouta, B.P. 1014, R.P. 10000 Rabat, Morocco. ${ }^{13}$ INFN - Laboratori Nazionali del Sud (LNS), Via S. Sofia 62, 95123 Catania, Italy. ${ }^{14}$ University Mohammed I, Laboratory of Physics of Matter and Radiations, B.P.717, Oujda 6000, Morocco. ${ }^{15}$ Nikhef, Science Park, Amsterdam, The Netherlands. ${ }^{16}$ Institute of Space Science, RO-077125 Bucharest, Măgurele, Romania. ${ }^{17}$ Universiteit van Amsterdam, Instituut voor Hoge-Energie Fysica, Science Park 105, 1098 XG Amsterdam, The Netherlands. ${ }^{18}$ INFN - Sezione di Roma, P.le Aldo Moro 2, 00185 Roma, Italy. ${ }^{19}$ Dipartimento di Fisica dell'Università La Sapienza, P.le Aldo Moro 2, 00185 Roma, Italy. ${ }^{20}$ LPHEA, Faculty of Science - Semlali, Cadi Ayyad University, P.O.B. 2390, Marrakech, Morocco. ${ }^{21}$ INFN - Sezione di Bologna, Viale Berti-Pichat 6/2, 40127 Bologna, Italy. ${ }^{22}$ INFN - Sezione di Bari, Via E. Orabona 4, 70126 Bari, Italy. ${ }^{23}$ Department of Computer Architecture and Technology/CITIC, University of Granada, 18071 Granada, Spain. ${ }^{24}$ Géoazur, UCA, CNRS, IRD, Observatoire de la Côte d'Azur, Sophia Antipolis, France. ${ }^{25}$ Université Paris-Sud, 91405 Orsay Cedex, France. ${ }^{26}$ Dipartimento di Fisica e Astronomia dell'Università, Viale Berti Pichat 6/2, 40127 Bologna, Italy. ${ }^{27}$ Laboratoire de Physique Corpusculaire, Clermont Université, Université Blaise Pascal, CNRS/IN2P3, BP 10448, F-63000 Clermont-Ferrand, France. ${ }^{28}$ LIS, UMR Université de Toulon, Aix Marseille Université, CNRS, 83041 Toulon, France. ${ }^{29}$ Dipartimento di Fisica dell'Università, Via Dodecaneso 33, 16146 Genova, Italy. ${ }^{30}$ Royal Netherlands Institute for Sea Research (NIOZ), Landsdiep 4, $1797 \mathrm{SZ}$ 't Horntje (Texel), the Netherlands. ${ }^{31}$ International Centre for Radio Astronomy Research - Curtin University, Bentley, WA 6102, Australia. ${ }^{32}$ Huygens-Kamerlingh Onnes Laboratorium, Universiteit Leiden, The Netherlands. ${ }^{33}$ Institut für Theoretische Physik und Astrophysik, Universität Würzburg, Emil-Fischer Str. 31, 97074 Würzburg, Germany. ${ }^{34}$ Dr. Remeis-Sternwarte and ECAP, Friedrich-Alexander-Universität Erlangen-Nürnberg, Sternwartstr. 7, 96049 Bamberg, Germany. ${ }^{35}$ Mediterranean Institute of Oceanography (MIO), Aix-Marseille University, 13288, Marseille, Cedex 9, France; Université du Sud Toulon-Var, CNRS-INSU/IRD UM 110, 83957, La Garde Cedex, France. ${ }^{36}$ INFN - Sezione di Catania, Via S. Sofia 64, 95123 Catania, Italy. ${ }^{37}$ Dpto. de Física Teórica y del Cosmos \& C.A.F.P.E., University of Granada, 18071 Granada, Spain. ${ }^{38}$ IRFU, CEA, Université Paris-Saclay, F-91191 Gif-sur-Yvette, France. ${ }^{39}$ INFN - Sezione di Napoli, Via Cintia 80126 Napoli, Italy. ${ }^{40}$ Museo Storico della Fisica e Centro Studi e Ricerche Enrico Fermi, Piazza del Viminale 1, 00184, Roma. ${ }^{41}$ INFN - CNAF, Viale C. Berti Pichat 6/2, 40127, Bologna. ${ }^{42}$ Institut Universitaire de France, 75005 Paris, France. ${ }^{43}$ Dipartimento di Fisica dell'Università Federico II di Napoli, Via Cintia 80126, Napoli, Italy. 